\title{
Primer reporte de Postharmostomum sp. (Witenberg, 1923) (Trematoda, Brachylaemidae) para Megalobulimus maximus (Gasteropoda, Megalobulimidae) en San Martín, Perú
}

\author{
First report of Postharmostomum sp. (Witenberg, 1923) (Trematoda, \\ Brachylaemidae) in Megalobulimus maximus (Mollusca, Gasteropoda) from \\ San Martín, Peru
}

Lidia Sánchez*, Patricia Salízar y Maria Morales

Presentado: 27/09/2004

Aceptado: $\quad 11 / 07 / 2005$

\section{Resumen}

Se colectaron 227 parásitos en estadío juvenil correspondientes al género Postharmostomum sp., Witenberg, 1923 (Trematoda: Digenea), localizados en la cavidad pericárdica de dos individuos del caracol terrestre Megalobulimus maximus colectados en el distrito de Juanjui, departamento de San Martín, Perú. Los ejemplares fueron depositados en la Colección Helmintológica y de Invertebrados Relacionados del Departamento de Protozoología Helmintología e Invertebrados Afines del Museo de Historia Natural de la Universidad Nacional Mayor de San Marcos (MUSM), Lima, Perú.

Palabras claves: Postharmostomum, Brachylaemidae, Megalobulimus, Mollusca, Juanjuí, Perú.

\section{Abstract:}

A total of 227 specimens of young adults of Postharmostomum sp., Witenberg, 1923 (Trematoda: Digenea) were collected from pericardial cavity of two indiviudals of the land snail Megalobulimus maximus from distrito of Juanjuí, San Martín. Parasits were deposited on Coleccion Helmintológica y de Invertebrados Relacionados, of the Museo de Historia Natural, Universidad Nacional Mayor de San Marcos (MUSM), Lima, Peru.

Keywords: Postharmostomum, Brachylaemidae, Megalobulimus, Mollusca, Juanjuí, Peru.

\section{Introducción}

En el Perú, los trabajos parasitológicos en caracoles terrestres son escasos, entre ellos podemos mencionar a Fasciola hepatica en el género Lymnaea, y Leucoclroridium en Succinea peruviana. Este es el primer estudio parasitológico donde se reporta al tremátodo Postharmostomum sp. (Brachylaeminae), en el caracol terrestre Megalobulimus maximus (Megalobulimidae), conocido comúnmente como «congompe», el cual comprende algunos de los mayores caracoles terrestres vivientes, que constituyen una importante fuente de alimentación en la región de la selva, siendo además una de las especies de mayor valor comercial.

\footnotetext{
*Departamento de Protozoología, Helmintología e Invertebrados Afines, Museo de Historia Natural, UNMSM. Lima - Perú. Av. Arenales 1256 Jesús María.

E-mail Lidia Sánchez: tano@qnet.com.pe
}

El género Postharmostomum sp. atrajo la atención de investigadores como Ulmer (1951a, 1951b) quien hizo un estudio del ciclo de vida de Postharmostomum helicis en el caracol terrestre Anguispira alternata, utilizando como hospedadores experimentales definitivos a ratones, conejos y pollos, lo que le permitió hacer una descripción de los caracteres morfológicos de cada uno de los estadios del parásito. Travassos y Kohn (1966) hacen una lista de los géneros que componen la superfamilia Brachylaimidae, dentro de los cuales mencionan a Postharmostomum gallinum y describen los principales caracteres morfológicos del género. Travassos et al. (1969) describen a Postharmostomum commutatum como un parásito de Gallus gallus. Schell (1985) en su recopilación de tremátodos de Norte América hace una pequeña descripción del ciclo de $P$. gallinum $y$ 


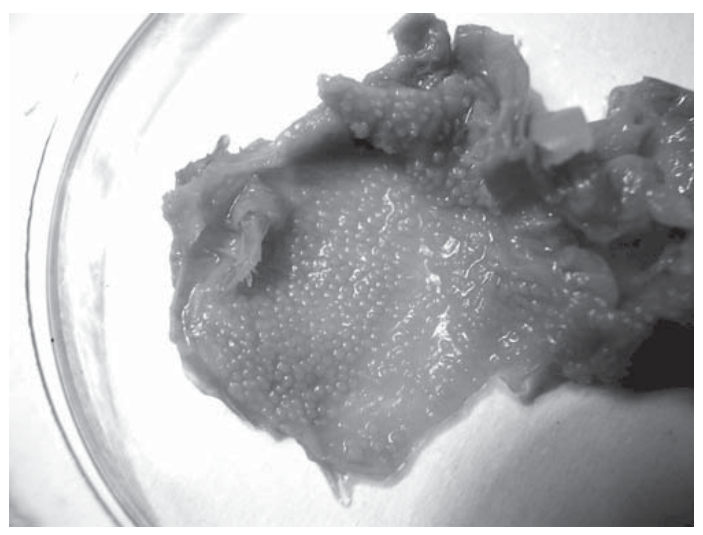

Figura 1. Tejido Pericárdico de Megalobulimus maximus mostrando el daño ocasionado por las metacercarias Postharmostomum sp.

P. helici. Amato y Bezerra (1993) estudiando el parasitismo del caracol terrestre Bradybaena similaris por P. gallinum realizan una infección experimental en pollos para determinar la especie. Thatcher (1993) en su publicación sobre tremátodos neotropicales describe a $P$. commutatum como una especie cosmopolita que parasita el intestino de aves. Pence (1995) hizo un estudio de Postharmostomiasis en pavos silvestres, donde describe a especímenes maduros e inmaduros de $P$. gallinum, dando un rango de medidas de longitud $(5,9$ y $11,3 \mathrm{~mm})$ y ancho (1,4 y 2,8 mm) para los especímenes adultos.

\section{Material y métodos}

Dos ejemplares de Megalobulimus maximus, provenientes del distrito de Juanjui, provincia de Mariscal Cáceres, departamento de San Martín, fueron recepcionados en el laboratorio para la evaluación respectiva. Los ejemplares fueron relajados en agua fría y posteriormente se les dio muerte y fijó en alcohol de $70 \%$, procediendo luego a realizar la disección. Las metacercarias halladas en la cavidad pericárdica fueron coloreadas con carmín semichron y montadas para su identificación, realizándose las mediciones correspondientes las que se dan en milimetros. El dibujo fue elaborado con ayuda del microscopio invertido.

\section{Resultados}

Se revisaron 2 ejemplares de Megalobulimus maximus hallándose dentro de su cavidad pericárdica un total de 227 parásitos en estadio juvenil los cuales fueron depositados en la Colección Helmintológica y de Invertebrados Relacionados del Departamento de Protozoología, Helmintología e Invertebrados Afines del Museo de Historia Natural de la Universidad de San Marcos (MUSM) con el número de ingreso 1730. También se observaron pequeñas granulaciones a todo lo largo de la pared de la cavidad pericárdica, que resultaron ser aglomeración de sales y fibras de tejido debido a la actividad alimentaria del parásito (Fig. 1).

Se hizo una comparación morfométrica (Tabla 1) para determinar el estadío de desarrollo de los parásitos. Esta primera comparación nos llevó a concluir que los parásitos encontrados eran juveniles, debiendo determinar en segunda instancia el tiempo de desarrollo de este estadío (Tabla 2). Los parámetros morfométricos hallados son muy próximos a los de 9 días de desarrollo de acuerdo a Ulmer (1951).

Tabla 1. Mediciones comparativas de Postharmostomum sp. adultos en milímetros (longitud x ancho).

\begin{tabular}{|c|c|c|c|c|c|c|}
\hline & $\begin{array}{c}\text { McIntosh } \\
1934\end{array}$ & $\begin{array}{c}\text { Miller } \\
1935\end{array}$ & $\begin{array}{c}\text { Robinson } \\
1949 a\end{array}$ & $\begin{array}{c}\text { Robinson } \\
1949 b\end{array}$ & Ulmer 1951 & $\begin{array}{c}\text { Presente } \\
\text { trabajo }\end{array}$ \\
\hline Cuerpo & $2,8 \times 1,4$ & $3,99 \times 1,32$ & $3,5 \times 2,0$ & $3,0 \times 1,38$ & $3,31 \times 1,46$ & $2,713 \times 1,231$ \\
\hline $\begin{array}{l}\text { Ventosa } \\
\text { Oral }\end{array}$ & $0,410 \times 0,500$ & $0,578 \times 0,497$ & $0,500 \times 0,580$ & $0,474 \times 0,535$ & $0,517 \times 0,532$ & $0,292 \times 0,354$ \\
\hline Faringe & $0,250 \times 0,250$ & - - & - - & $0,242 \times 0,250$ & $0,236 \times 0,266$ & $0,249 \times 0,242$ \\
\hline Acetábulo & $0,420 \times 0,450$ & $0,487 \times 0,487$ & $0,460 \times 0,560$ & $0,365 \times 0,432$ & $0,458 \times 0,458$ & $0,354 \times 0,359$ \\
\hline
\end{tabular}

Fuente: Ulmer, 1951a 
Tabla 2. Mediciones comparativas de Postharmostomum sp. en milímetros (longitud x ancho)

\begin{tabular}{lcccc}
\hline & \multicolumn{3}{c}{ Ulmer, 1951 (adultos) } & \\
\cline { 2 - 4 } & 9 dias & 40 dias & 100 dias & Sánchez (promedio) \\
\hline Cuerpo & $2,542 \times 0,961$ & $4,581 \times 1,848$ & $4,692 \times 2,069$ & $2,713 \times 1,231$ \\
Ventosa & $0,399 \times 0,428$ & $0,517 \times 0,605$ & $0,650 \times 0,724$ & $0,292 \times 0,354$ \\
Oral & & & & \\
Faringe & $0,177 \times 0,240$ & $0,266 \times 0,310$ & $0,296 \times 0,370$ & $0,249 \times 0,242$ \\
Acetábulo & $0,340 \times 0,340$ & $0,487 \times 0,540$ & $0,605 \times 0,650$ & $0,354 \times 0,359$ \\
\hline
\end{tabular}

Fuente: Ulmer, 1951b

\section{Taxonomía y Descripción}

Clase Trematoda Rudolphi, 1808.

ORDEN BRACHYLAEMOIDEA NOM. N.

Familia Brachylaemidae Joyeux \& Foley, 1930.

Subfamilia Brachylaeminae Joyeux \& Foley, 1930

Postharmostomum sp. Witenberg, 1923

(Fig. 2)

Los juveniles presentan un cuerpo alargado, fusiforme, con 1,106 a 3,094 mm x 0,966 a $1,498 \mathrm{~mm}$. Cutícula lisa. Ventosa oral subterminal, con 0,126 a $0,420 \mathrm{~mm} \times 0,280$ a

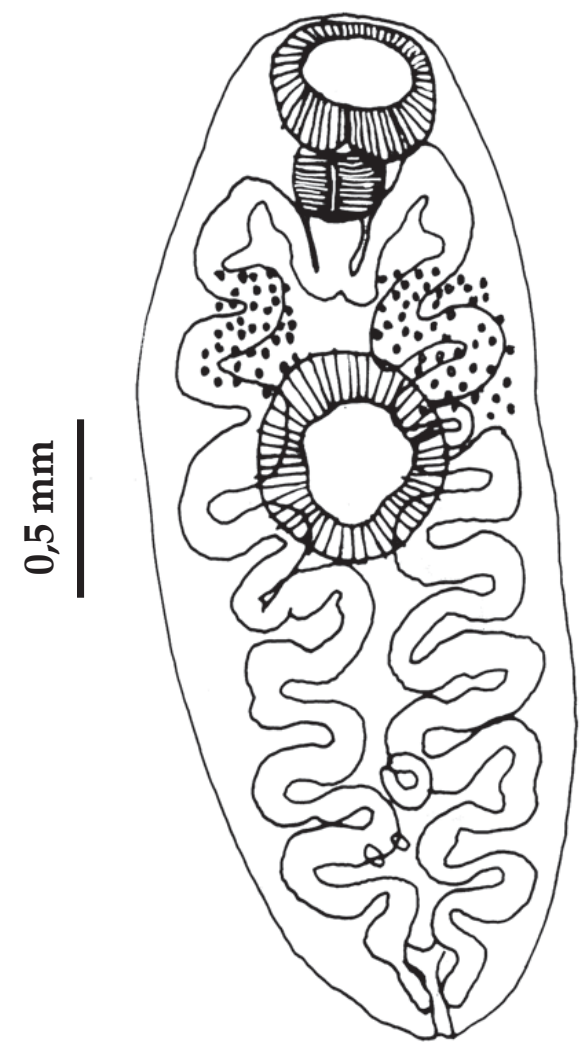

Figura 2. Metacercaria de Postharmostomum sp.
$0,420 \mathrm{~mm}$. Acetábulo mediano, pre-ecuatorial, con 0,238 a 0,434 x 0,252 a $0,448 \mathrm{~mm}$. Prefaringe ausente. Faringe con 0,168 a 0,280 x 0,210 a 0,294 mm. Esófago muy corto. Asas intestinales con muchas sinuosidades, cuyos campos casi se tocan en la línea media del cuerpo, terminando muy próximos al extremo caudal, sin unirse. Presenta un complejo genital desarrollado, constituido por primordios genitales ubicados en el cuarto posterior del cuerpo y conductos asociados fácilmente distinguibles. En el extremo anterior del acetábulo se puede observar primordios del útero. Se observan trazos del sistema excretor, constituido por una vejiga excretora que termina posteriormente con el poro excretor $y$ se prolonga anteriormente en dos conductos.

\section{Discusión}

La característica principal para determinar a los tremátodos juveniles encontrados en Megalobulimus maximus como género Postharmostomum sp., es la presencia de asas intestinales muy contorneadas que no se observan en otros géneros de la misma familia. Estas metacercarias podrían confundirse con un estadío enquistado de la especie Brachylaima virginianum, descrita por Ulmer (1952) cuyas asas intestinales son tan sinuosas como las de Postharmostomum sp., pero en cuyo estado libre estas asas intestinales se hacen rectas, en cambio los ejemplares del género Postharmostomum sp., mantienen la forma muy sinuosa de las asas intestinales en su forma no enquistada. Además la posición del acetábulo varía en la metacercaria enquistada de Brachylaima virginianum, ob- 
servándose muy próxima a la faringe, mientras que en Postharmostomum sp. esta posición es pre-ecuatorial. Otra característica considerada, fue la presencia de un esófago corto.

La comparación con caracteres morfométricos presentados por los diferentes autores nos ayudaron a determinar el estadío de los tremátodos estudiados.

La ubicación del tremátodo en el hospedero es un carácter diferencial para el género, en este caso se les encontró en la cavidad pericárdica, coincidiendo con los reportes consultados.

El daño que ocasiona este parásito en el tejido que envuelve la cavidad pericárdica es evidente y su proporción está en relación directa con el número de individuos recolectados.

Para la determinación de la especie se hace necesario completar el ciclo biológico con los posibles hospedadores definitivos: roedores y/o aves silvestres y domésticas, siendo esto motivo de un trabajo posterior.

\section{Literatura citada}

Amato, S. y J. Becerra. 1993. Parasitismo natural de Bradybaena similaris (Férussac, 1821) por Postharmostomum gallinum Witenberg, 1923. CABI Online Abstract Journals Data Service. www.cabsubsets.org

Pence, D. 1995. Postharmostomiasis in wild turkeys in New Mexico, CABI Online Abstract Journals Data Service. www.cabsubsets.org

Ramírez, R. 1992. Esporocisto de Leucochloridium (Trematoda, Leucochloridiidae) en Succinea peruviana (Mollusca, Succineidae). Anales Inst. Biol. Univ. Nac. Autón. México, Ser. Zool. 63(2): 173 - 177.

Schell, S. 1985. Trematodes of North America, UPI editores, Idaho, $263 \mathrm{p}$.

Thatcher, V. 1993. Trematódeos Neotropicais, INPA editores, Brasil, $553 \mathrm{p}$.

Travassos, L.; K. KOHN. 1966. Lista dos gêneros incluídos na superfamilia Brachylaemoidea. Mem. Inst. Oswaldo Cruz, 64 (fasc. único):1125.

Travassos L.; L. F. Teixeira De Freitas y A. Kohn 1969. Trematódeos do Brasil. Mem. Inst. Oswaldo Cruz. 67(1):623-264.

Ulmer, M.1951a. Postharmostomum helicis (Leidy, 1847) Robinson 1949, (Trematoda), its life history and a revision of the subfamily Brachylaeminae.Trans. Amer. Micros. Soc.70 (3):189-238.

Ulmer, M.1951b. Postharmostomum helicis (Leidy, 1847) Robinson 1949, (Trematoda), its life history and a revision of the subfamily Brachylaeminae.Trans. Amer. Micros. Soc. 70 (4):319-347.

Ulmer, M. 1952. Morphologial features of Brachylaima virginianum metacercarie (Trematoda: Brachylaimatidae), and migration route or cercariae in the second intermediate host, Journal of Science 27 (3):91-103. 